\title{
Real-Time Integrated Limited-Stop and Short-Turning Bus Control with Stochastic Travel Time
}

\author{
Hu Zhang, Shuzhi Zhao, Yang Cao, Huasheng Liu, and Shidong Liang \\ College of Transportation, Jilin University, Changchun 130022, China \\ Correspondence should be addressed to Yang Cao; yang_cao@jlu.edu.cn
}

Received 12 March 2017; Revised 20 May 2017; Accepted 6 June 2017; Published 12 July 2017

Academic Editor: Seungjae Lee

Copyright (C) $2017 \mathrm{Hu}$ Zhang et al. This is an open access article distributed under the Creative Commons Attribution License, which permits unrestricted use, distribution, and reproduction in any medium, provided the original work is properly cited.

In a traditional transit system, passenger arrival time and bus running time are typically random and uncoordinated. This randomness gives the appearance of unbalanced passenger demand and unreliable transit services. Therefore, this paper proposes a real-time control method for bus routes. In our method, buses skip some stations and turn back at appropriate stations, in order to balance passenger demand along the bus route and improve the overall transit service. Our real-time control method considers the typical changes in passenger demand and the stochastic travel time of buses. In this paper, the number of controlled vehicles at any given time is determined, and the bus holding time at the turn-back station is adopted. When implemented correctly, the optimal scheme indicates which stations should be skipped, where it is suitable for buses to turn back, and how long the holding time should be at turn-back stations, which in turn will minimize the total cost of a transit system. This paper formulates such an integrated strategy, presents the solution method of the formulation, and proves the validity of the real-time control method.

\section{Introduction}

In virtually any transit system, passenger demand is unbalanced along the bus route. Also, the time it takes the bus to travel the designated route cannot be consistent in practice, because of road traffic situations and driver operating habits, which combined to make the change of travel time stochastic. Various levels of passenger demand at different stations and stochastic travel time can decrease the transit service quality when buses run along their same routes with the same operation techniques employed all day, every day. Without additional strategies, public resources cannot be fully utilized. In addition, the variety of factors mentioned above and the stochastic changes brought about will create a "bus bunching" effect and increase passenger waiting time. Thus, a real-time control strategy should be implemented among the transit system routes as a means to avoid confusion surrounding bus operations and to improve the level of service.

Lately, ITS technologies have been widely applied to transit system operations. These technologies include automatic vehicle location (AVL) or automatic vehicle identification (AVI) systems, as well as automatic passenger counters
(APC). These technologies can effectively assure that vehicle movements and passenger flows can be fairly and accurately predicted over a short time interval. With regard to realtime control strategies, many studies have been conducted on bus holding strategies. Turnquist and Blume [1] found that holding strategies could be used to increase service regularity. Abkowitz and Lepofsky [2] proposed holding buses at appropriate stopping points and then dispatching them when a minimum headway time could be achieved, thus minimizing the total passenger waiting time. Considering safety headways, Eberlein et al. [3] set a rolling horizon and presented a formulation to minimize the total waiting time. Their research results indicated that holding could reduce dwelling time and interstation stopping time. Fu and Yang [4] argued, however, that large headway variations would increase the waiting time. As such, they proposed holding buses at a single station, in order to minimize headway variations. Delgado et al. [5] proposed an integrated holding and boarding limits strategy. This strategy does not allow passengers to board buses once the strategic limit has been reached, even when the bus in question has sufficient capacity to take additional passengers. 
Meanwhile, operational strategies (whereby buses do not need to serve all stations along the route) are controlled through a real-time method. Various operational strategies have been proposed, including short-turning, deadheading, and limited-stop services [6-8]. To increase the speed of a subway service, Suh et al. [9] formulated a stop-skipping strategy on a rail system. This formula used an OD matrix, the distances between stations, and departure intervals. Fu et al. [10] proposed a real-time control strategy that would provide a limited-stop line every second trip, thus minimizing total waiting time costs. In addition, a rolling time horizon approach was used to define the stops which could be skipped. Sun and Hickman [11] also presented a stopskipping strategy. However, their strategy merely restricted the boarding of passengers at the skipped stations where passengers were still allowed to alight. Sidi et al. [12] considered a disruption strategy. Sidi et al. proposed a multiobjective optimization approach to determine which stations should be skipped, as well as the departure time of the controlled buses. Cortés et al. [13] and Sáez et al. [14] proposed an integrated stop-skipping and holding strategy, which was intended to minimize waiting time costs and the passengers' invehicle time. In these strategies, GAs were used to solve the formulation. Yu et al. [15] presented a partway deadheading strategy to improve transit service. This method determines the controlled vehicles by a reliability assessment of further transit services. Muñoz et al. [16] compared several of the mechanisms which had already been proposed in various studies and then estimated the validity of those studies.

Obviously, some of these previous studies indicate that real-time control strategies can increase service regularity and reduce the overall cost of a transit system. This paper proposes and reveals a real-time integrated limited-stop and short-turn strategy. Our limited-stop strategy allows buses to skip some stations, in order to better serve high-demand areas and times and thus can balance the demand distribution along the bus route. The short-turn strategy is a useful means by which to reduce bus running time. In this strategy, buses turn back to service the return direction of their route, thus creating a shorter cycle. What is more, in order to reflect the reality of the transit system, the impact of stochastic travel time is considered. In this paper, the holding time at the turnback station is determined, and the numbers of vehicles to be controlled at any given time are calibrated. The results of our test indicate that our real-time integrated strategy can reduce the total operating cost. In addition, implementing the holding strategy at turn-back stations can have a significantly positive effect on the overall result. Following this introduction, Section 2 describes our integrated strategy and establishes formulations to express the arrival time, departure time, and holding time of buses. Section 3 establishes the objective function to minimize the total transit system cost. Section 4 presents our solution methods to solve the objective function. Section 5 determines the number of controlled vehicles and tests the real-time integrated strategy, comparing our results to other strategies. Finally, Section 6 presents our conclusions.

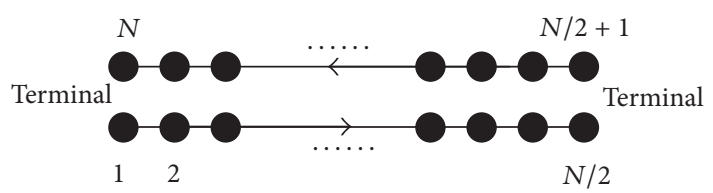

FIgURE 1: The transit route.

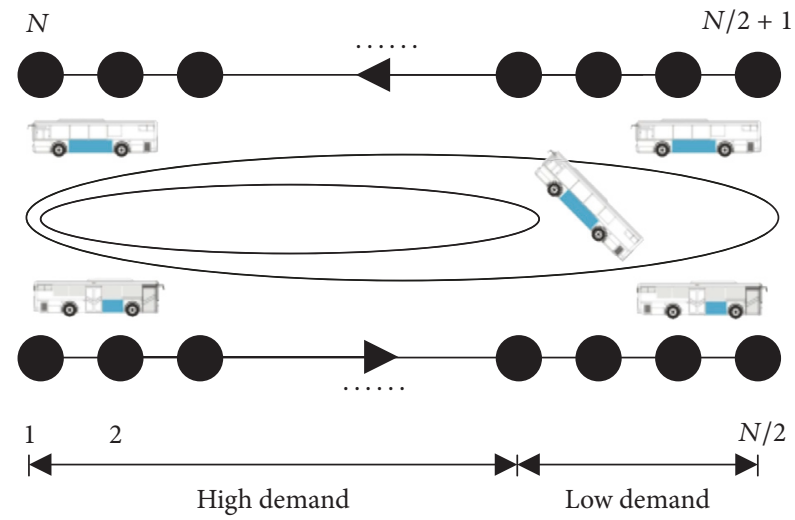

FIGURE 2: The integrated limited-stop and short-turning strategy.

\section{Problem Setting}

2.1. Stop-Skipping Strategy Description. This paper considers a transit route with $N$ stops, as shown in Figure 1. Buses are dispatched from terminals $1(N)$ and $N / 2+1(N / 2)$, according to a given schedule. In one direction, buses operate from Station 1 to Station $N / 2$. In the other directions, station $N / 2+1$ and station $N$ are the starting and ending terminals, respectively. We assume the largest passenger demand is close to station $1(N)$.

The integrated limited-stop and short-turning strategy is different from the regular transit service, as shown in Figure 2. Buses use the integrated limited-stop and shortturning strategy service high-demand stations and skip the low demand stations. Meanwhile, some buses may turn back before they reach the low demand station, if the stations near terminals $N / 2+1(N / 2)$ in both directions have low demand. Stop-skipping and turn-back services are therefore able to supply more buses for high-demand stations.

An integrated limited-stop and short-turning strategy can allow buses to serve stations with higher demand. Considering that passenger demand cannot always be high before the turn-back station, the buses using the integrated strategy will firstly operate a limited-stop service, in order to reduce the riding time of in-vehicle passengers. Secondly, under the short-turning strategy, buses will turn back to skip those stations with low demand when driving in both directions. This in turn will, to a great extent, reduce bus travel time. Since passenger transfers must be considered, a fleet of buses which operate an all-stop service will be utilized in our integrated strategy. The decisions regarding which stations can be skipped and whether or not a turn-back action should be used will be controlled in real-time. 
A real-time control problem is dynamic in nature. We consider that there is a group of $m$ controlled vehicle trips at any given time. There are $m-1$ vehicles to be controlled with the integrated strategy. Meanwhile, the $m$ th vehicle is also controlled and just operates with normal service. Those controlled buses are denoted as $i, i+1, \ldots, i+m-1$. Thus, the buses $i-1$ and $i+m$ will service all stations. In other words, we consider a rolling horizon of size $m$.

2.2. The Integrated Limited-Stop and Short-Turning Formulation. A traditional transit system follows two identical processes in the movement of buses and passengers. Operating on a given route, buses will pass through a section of roadway between adjacent stations and then stop at each station to set down some passengers and pick up others, before exiting the station. If a station is determined as suitable for being skipped, however, the dwelling time that would have taken place at the skipped station will disappear, and the bus will run directly to the next station. On any given transit service trip, passengers will arrive at a station and then wait for a bus. They will then board and travel in the bus that is running to their destination. An integrated limited-stop and shortturning strategy can reduce running time of buses, in-vehicle time of passenger, and waiting time of passenger at serving stations. However, this type of service could also increase passenger waiting time at skipping stations. Therefore, the transit service should be optimized, in order to reduce the total costs of buses and the costs in time to passengers.

The process whereby bus $i$ operates from station $j-1$ to station $j$ is such that bus $i$ makes an accelerated departure from station $j-1$ and then travels in the section between station $j-1$ and station $j$. The bus then decelerates to arrive at station $j$. The arrival time of bus $i$ at station $j$ can be calculated as follows:

$$
t_{i, j}^{A}=t_{i, j-1}^{D}+t_{\mathrm{ac}} \cdot y_{i, j-1}+t_{j-1, j}^{R}+t_{\mathrm{de}} \cdot y_{i, j} .
$$

Moreover, note that if buses turn back at station $j^{\prime}(N-$ $\left.j^{\prime}+1\right)$, the above formula cannot express the arrival time of buses at turn-back station $N-j^{\prime}+1$. Departing station $j^{\prime}$, buses will travel directly to station $N-j^{\prime}+1$, but they will not stop at station $j^{\prime}+1$. In order to avoid bus bunching as much as possible (and thus improve the transit service), a bus arriving at station $N-j^{\prime}+1$ should maintain a proper distance between itself, the previous bus, and the next bus. Therefore, a holding time $\left(t_{i, j^{\prime}}^{h}\right)$ should be introduced for the turn-back bus at station $j^{\prime}$, as shown in Figure 3. The arrival time of buses at turn-back station $N-j^{\prime}+1$ can be expressed by

$$
t_{i, N-j^{\prime}+1}^{A}=t_{i, j^{\prime}}^{D}+t_{i, j^{\prime}}^{h}+t_{j^{\prime}, N-j^{\prime}+1}^{R} .
$$

When bus $i$ turns back and arrives at station $N-j^{\prime}+1$, the previous bus and the next bus are denoted as $i^{\prime}$ and $i^{\prime}+1$, respectively. Also, the previous bus $i^{\prime}$ can be determined by

$$
\begin{aligned}
& \left(t_{i, j^{\prime}}^{D}+t_{j^{\prime}, N-j^{\prime}+1}^{R}\right)-t_{i^{\prime}, N-j^{\prime}+1}^{A} \\
& \quad=\min _{l=i-1, i-2, \ldots}\left[\left|\left(t_{i, j^{\prime}}^{D}+t_{j^{\prime}, N-j^{\prime}+1}^{R}\right)-t_{l, N-j^{\prime}+1}^{A}\right|\right] .
\end{aligned}
$$

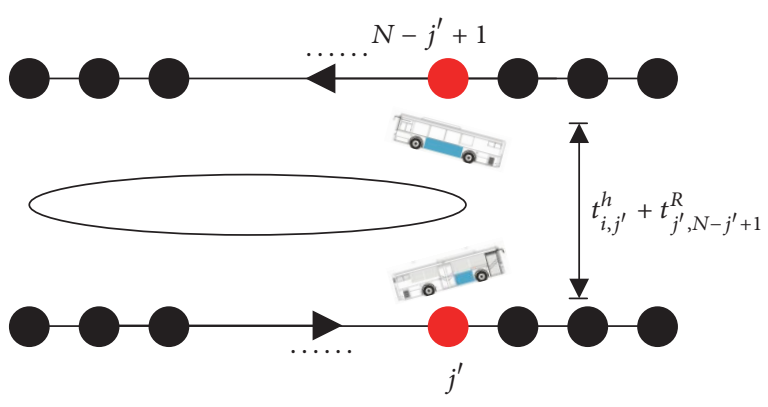

FIgURE 3: Process of buses turning back.

The hold time can be calculated by

$$
t_{i, N-j^{\prime}+1}^{A}-t_{i^{\prime}, N-j^{\prime}+1}^{A}=t_{i^{\prime}+1, N-j^{\prime}+1}^{A}-t_{i, N-j^{\prime}+1}^{A} .
$$

Namely,

$$
t_{i, j^{\prime}}^{h}=\frac{1}{2}\left(t_{i^{\prime}, N-j^{\prime}+1}^{A}+t_{i^{\prime}+1, N-j^{\prime}+1}^{A}-2 t_{i, j^{\prime}}^{D}-2 t_{j^{\prime}, N-j^{\prime}+1}^{R}\right) .
$$

If bus $i$ turns back at station $j^{\prime}\left(N-j^{\prime}+1\right)$, the bus queue will be $\left\{\ldots, i+1, i-1, i-2, \ldots, i^{\prime}+1, i, i^{\prime}, \ldots\right\}$. In order to facilitate the calculation of bus queues, the bus queue between $i-1$ and $i^{\prime}+1$ can be renumbered as $\left\{\ldots, i, i-1, i-2, \ldots, i^{\prime}+\right.$ $\left.2, i^{\prime}+1, i^{\prime}, \ldots\right\}$.

After passengers board and alight from a bus, that bus departs from the station. The departure time of bus $i$ from station $j$ can be expressed by

$$
t_{i, j}^{D}=t_{i, j}^{A}+t_{i, j}^{E} .
$$

Therefore, to accurately determine the departure time $\left(t_{i, j}^{D}\right)$, we should compute the dwelling time of buses at the relevant station. Dwelling time is closely related to the number of passengers who board and alight from the bus. Since boarding and alighting behaviors are conducted simultaneously, bus holding time will naturally depend on boarding and alighting time. The boarding time is the number of passengers boarding the bus $i\left(N_{i, j}^{+}\right)$multiplied by the average boarding time per passenger $(\alpha)$. Also, the alighting time is the number of passengers alighting from the bus $i\left(N_{i, j}^{-}\right)$multiplied by the average alighting time per passenger $(\beta)$. The dwelling time of bus $i$ at station $j$ can therefore be calculated as follows:

$$
t_{i, j}^{E}=\max \left[\alpha \cdot N_{i, j}^{+}, \beta \cdot N_{i, j}^{-}\right] .
$$

Assume that an origin-destination trip matrix can be given. Passengers will select the bus which serves their origin and destination stations. In one direction from Station 1 to Station $N / 2$, the arrival rates of boarding and alighting passengers at station $j$ are equal to $\sum_{k=j+1}^{N / 2} \lambda_{i, j \rightarrow k} \cdot y_{i, j} \cdot y_{i, k}$ and $\sum_{k=1}^{j-1} \lambda_{i, k \rightarrow j} \cdot y_{i, k} \cdot y_{i, j}$. And, in the other direction, $\sum_{k=j+1}^{N} \lambda_{i, j \rightarrow k}$. $y_{i, j} \cdot y_{i, k}$ and $\sum_{k=N / 2+1}^{j-1} \lambda_{i, k \rightarrow j} \cdot y_{i, k} \cdot y_{i, j}$ express the arrival rates of boarding and alighting passengers at station $j$. The 
numbers of boarding and alighting passengers at station $j$ can be calculated as follows:

$$
\begin{aligned}
& N_{i, j}^{+}=W_{i, j} \cdot \sum_{k=j+1}^{N / 2} \lambda_{i, j \rightarrow k} \cdot y_{i, j} \cdot y_{i, k}, \\
& N_{i, j}^{-}=\sum_{k=1}^{j-1}\left(\lambda_{i, k \rightarrow j} \cdot y_{i, k} \cdot y_{i, j} \cdot W_{i, k}\right) .
\end{aligned}
$$

Considering that buses may skip some stations, bus headway is not equal to the average waiting time of passengers $\left(W_{i, j}\right)$. If bus $i-1$ (ahead) is determined to skip station $j$, passenger waiting time at station $j$ will correspondingly expand. The average passenger waiting time can be expressed by

$$
\begin{aligned}
& W_{i, j} \\
& = \begin{cases}t_{i, j}^{D}-t_{i-1, j}^{D}, & y_{i-1, j}=1, \\
t_{i, j}^{D}-t_{i-2, j}^{D}, & y_{i-1, j}=0, \quad y_{i-2, j}=1, \\
t_{i, j}^{D}-t_{i-3, j}^{D}, & y_{i-1, j}+y_{i-2, j}=0, \quad y_{i-3, j}=1, \\
\vdots & \\
t_{i, j}^{D}-t_{i-k, j}^{D}, & y_{i-1, j}+y_{i-2, j}+\cdots+y_{i-k+1, j}=0, \quad y_{i-k, j}=1 .\end{cases}
\end{aligned}
$$

\section{Optimization Model}

The integrated limited-stop and short-turning strategy has the objective of reducing the total cost of a transit system. The cost of buses includes the sum of the running time cost between adjacent stations and the dwelling time cost at stations along the bus route. The cost to passengers includes the waiting time cost and in-vehicle time cost.

Regarding the cost of buses, the running time and the dwelling time of the $m$ group of controlled vehicle trips at any given time are calculated as

$$
C_{B}=c_{b} \cdot \sum_{i=1}^{m} \sum_{j=2}^{N}\left(t_{i, j}^{D}-t_{i, j-1}^{D}\right)
$$

Regarding the waiting time cost to passengers, the realtime strategy of each bus will be shown to passengers by means of an electronic bus stop notice board. The information posted on this board could also be conducive to customers selecting the proper bus. The waiting time costs to passengers are calculated as

$$
\begin{gathered}
C_{W}=c_{w} \cdot \sum_{i}^{m}\left(\sum_{j=1}^{N / 2-1} \sum_{k=2}^{N / 2} W_{i, j} \cdot N_{i, j}^{+}\right. \\
\left.+\sum_{j=N / 2}^{N-1} \sum_{k=N / 2+1}^{N} W_{i, j} \cdot N_{i, j}^{+}\right)=c_{w}
\end{gathered}
$$

$$
\begin{aligned}
& \cdot \sum_{i}^{m}\left(\sum_{j=1}^{N / 2-1} \sum_{k=2}^{N / 2} W_{i, j}^{2} \cdot \sum_{k=j+1}^{N / 2} \lambda_{i, j \rightarrow k} \cdot y_{i, j} \cdot y_{i, k}\right. \\
& \left.+\sum_{j=N / 2}^{N-1} \sum_{k=N / 2+1}^{N}{W_{i, j}}^{2} \cdot \sum_{k=j+1}^{N} \lambda_{i, j \rightarrow k} \cdot y_{i, j} \cdot y_{i, k}\right) .
\end{aligned}
$$

As regards the in-vehicle travel time cost to passengers, the in-vehicle passengers will skip several stations, which will shorten their trip time. The total in-vehicle time costs to passengers taking $m$ buses can be expressed by

$$
\begin{aligned}
C_{I} & =c_{i} \cdot \sum_{i}^{m}\left[\sum_{j=1}^{N / 2-1} \sum_{k=2}^{N / 2}\left(t_{i, k}^{A}-t_{i, j}^{D}\right) \cdot N_{i, j}^{+}\right. \\
& \left.+\sum_{j=N / 2}^{N-1} \sum_{k=N / 2+1}^{N}\left(t_{i, k}^{A}-t_{i, j}^{D}\right) \cdot N_{i, j}^{+}\right]=c_{i} \\
& \cdot \sum_{i}^{m}\left[\sum_{j=1}^{N / 2-1} \sum_{k=2}^{N / 2}\left(t_{i, k}^{A}-t_{i, j}^{D}\right) \cdot W_{i, j} \cdot \sum_{k=j+1}^{N / 2} \lambda_{i, j \rightarrow k} \cdot y_{i, j} \cdot y_{i, k}\right. \\
& \left.+\sum_{j=N / 2}^{N-1} \sum_{k=N / 2+1}^{N}\left(t_{i, k}^{A}-t_{i, j}^{D}\right) \cdot W_{i, j} \cdot \sum_{k=j+1}^{N} \lambda_{i, j \rightarrow k} \cdot y_{i, j} \cdot y_{i, k}\right] .
\end{aligned}
$$

The above models can be used to calculate the total costs of a transit system in the two directions of any given bus route. In order to ensure that the real-time integrated strategy can reduce the total costs, the objective function that minimizes the sum of the bus cost, in-vehicle time cost, and waiting time cost is established. The function is shown as follows:

$$
\min C=C_{B}+C_{W}+C_{I}
$$

Subject to: $y_{i, j}=\{0,1\}, \quad j=1,2, \ldots, j^{\prime}, x_{i, j^{\prime}}=1$,

$$
\begin{aligned}
& y_{i, 1}=y_{i, N}=1, \quad i=1,2, \ldots, m, \\
& y_{i, N / 2}=y_{i, N / 2+1}=1,
\end{aligned}
$$

$$
\begin{aligned}
& y_{m, j}=1, \quad j=1,2, \ldots, N, \\
& x_{i, j^{\prime}}=\{0,1\}, \quad j^{\prime} \in J^{\prime}, \\
& t_{i+1, j}^{A}-t_{i, j}^{D} \geq H_{\min }, \\
& \quad i=1,2, \ldots, m ; j=1,2, \ldots, N .
\end{aligned}
$$

Equations (14)-(18) are constraints of the objective function, which is used to improve the solution methods process. Station 1 and station $N$ cannot be skipped, and the output of the integrated strategy is the stop-skipping scheme between station 1 and station $j^{\prime}$. Whether or not bus $i$ turns back at station $j^{\prime}$ is indicated by different variables $x_{i, j^{\prime}}$; that is, $x_{i, j^{\prime}}=$ 1 indicates that the bus $i$ will turn back at station $j^{\prime}$; otherwise, $x_{i, j^{\prime}}=0$ means turn-back action of bus $i$ does not appear 


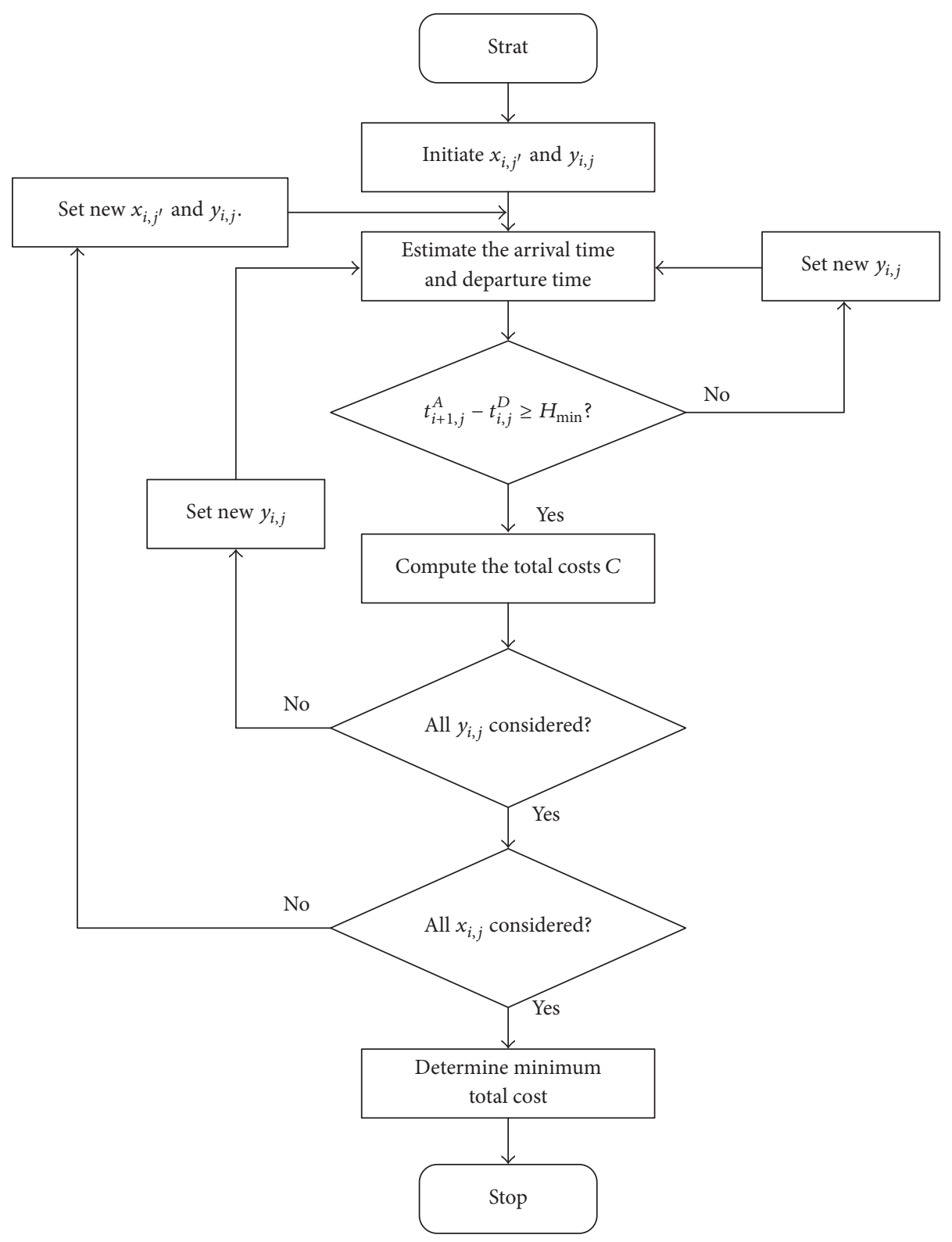

FIGURE 4: Solution algorithm.

at station $j^{\prime}$. Equation (19) is a constraint for the objective function. In the process of optimization, it can filter out the schemes that cause the smaller headway than the minimum headway. Remaining schemes are to ensure that the headway between the previous bus and the next bus is larger than the minimum headway, in order to avoid vehicle bunching and to guarantee a high-quality transit service.

3.1. Solution Methods. The optimization model in the previous section is intended to find and output the discrete optimization variables of appropriate limited-stop stations and short-turn stations, which is a problem of nonlinear 0,1 . A solution algorithm for the optimization model is required to solve the problem and obtain the optimum solution. Thus, an exhaustive search method was selected. We used the
Monte Carlo simulation method to output the stochastic travel time.

In the solution algorithm, an exhaustive search algorithm is programmed to find the short-turn station and proper skipping stations. The optimal scheme is as shown in (20), where values of the stations between short-turn station $j^{\prime}$ and $N-j^{\prime}+1$ are set as zero and $x_{i, j^{\prime}+N / 2}$ is equal to $x_{i, j^{\prime}}$. Values of $x_{i, j^{\prime}}$ and $y_{i, j}$ are constantly reset to find the optimal scheme. Figure 4 illustrates the solution algorithm for the above objective function, as follows:

$$
\begin{gathered}
{\left[y_{i, 2}, y_{i, 3}, \ldots, y_{i, j}, \ldots, y_{i, j^{\prime}-1}, x_{i, j^{\prime}}, 0,0, \ldots, 0, x_{i, N-j^{\prime}+1},\right.} \\
\left.y_{i, N-j^{\prime}+2}, \ldots, y_{i, N}\right], \quad j^{\prime} \in\left(2, \frac{N}{2}\right), j \in\left(2, j^{\prime}\right) .
\end{gathered}
$$




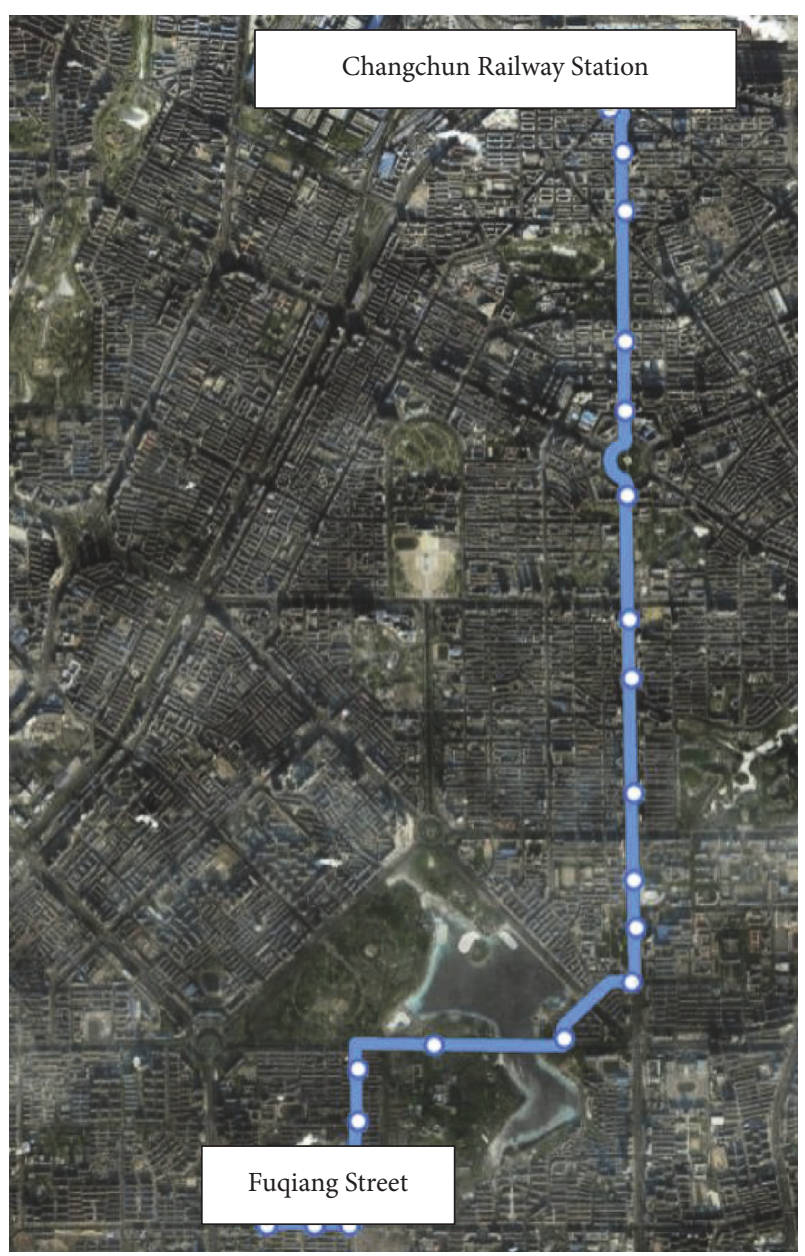

FIgURE 5: Configuration of transit route.

3.2. Numerical Test. The integrated limited-stop and shortturn strategy in this paper was tested on a bus route in Changchun City in China, as shown in Figure 5. Route number 6 (with 20 stations and $9.9 \mathrm{~km}$ in each direction) runs between Fuqiang Street and Changchun Railway Station. The departure interval is 5 minutes. The running time between the origin and destination of the bus route is scheduled to be 29 minutes. The variance of travel time between the adjacent stations is $10 \%$ of the total travel time. The starting station (Fuqiang Street) is located uptown, and the station at the end of the route (Changchun Railway Station) is downtown. During the early peak time period, passengers mainly flow to the downtown area. At this time, the average passenger demand shows unbalanced distribution, as shown in Figure 6. Assuming that the passengers boarding at station $j$ will alight at relatively even rates at the following stations, the origin-destination trip matrix can then be obtained. Difference of boarding and alighting demands between stations reflects the unbalance of passenger demand on the bus route. The headway and travel time are, respectively, five minutes and $19 \mathrm{~km} / \mathrm{h}$. Average passenger boarding and alighting time are, respectively, 5.2 seconds and 4.4 seconds. Time values are

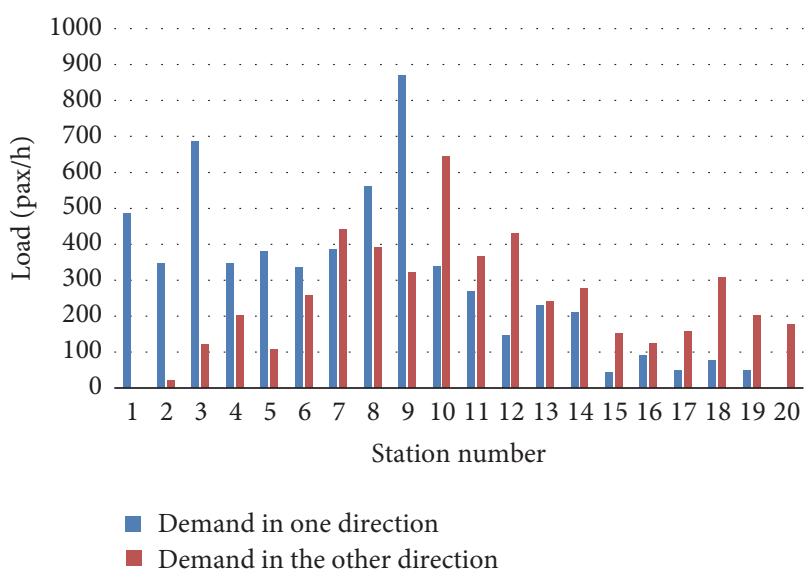

FIGURE 6: Average passenger demand in both directions during the early peak period.

assumed to be $\$ 15 /$ pax-h for waiting time, $\$ 10 /$ pax-h for invehicle time, and $\$ 50 /$ veh-h for running time.

We applied the mathematical software MATLAB R2011a to test the integrated limited-stop and short-turn strategy. This software can determine the bus arrival departure time, and the waiting and in-vehicle time of passengers, as well as the total cost of a transit system.

\section{Determination of the Number of Controlled Vehicle Trips}

Before calculating the objective function, the number of controlled vehicle trips at any one time $(\mathrm{m})$ should first be determined. This will have an effect on the results of the realtime controls. In previous studies $[10,17,18]$, the use of a controlled vehicle with stop-skipping strategy, plus a normal vehicle, was suggested to service all stations. This strategy is commonly used. Also, this method of using a controlled vehicle and a normal vehicle has a specific limitation, namely, that the proportion of controlled vehicles must be less than $50 \%$ of the total number of vehicles in use. In such cases, the number of controlled vehicles is so small that it cannot achieve the objective of balancing passenger demand. Also, the number of controlled vehicles was set by the authors of these studies to specific values. As such, if the number of controlled vehicles is too big, the timeliness and effectiveness of the real-time strategy may decline, due to potential inaccuracies in the realtime parameters. Therefore, we conducted an examination to determine the appropriate number of controlled vehicle trips at a given time $(m)$. Figure 7 shows the total costs of each controlled cycle during peak demand hours, with the number of controlled vehicle trips at one time varied. In addition, the total cost of each controlled cycle has been converted to costs per hour, for the convenience of comparison.

We can see that the number of controlled vehicles can influence the total cost of each controlled cycle. We can also clearly see that if the number of controlled vehicles is too low or too high, the total cost will increase. Specifically, when the number of controlled vehicles is equal to 4 , the total cost of each controlled cycle is the lowest. This finding means that 


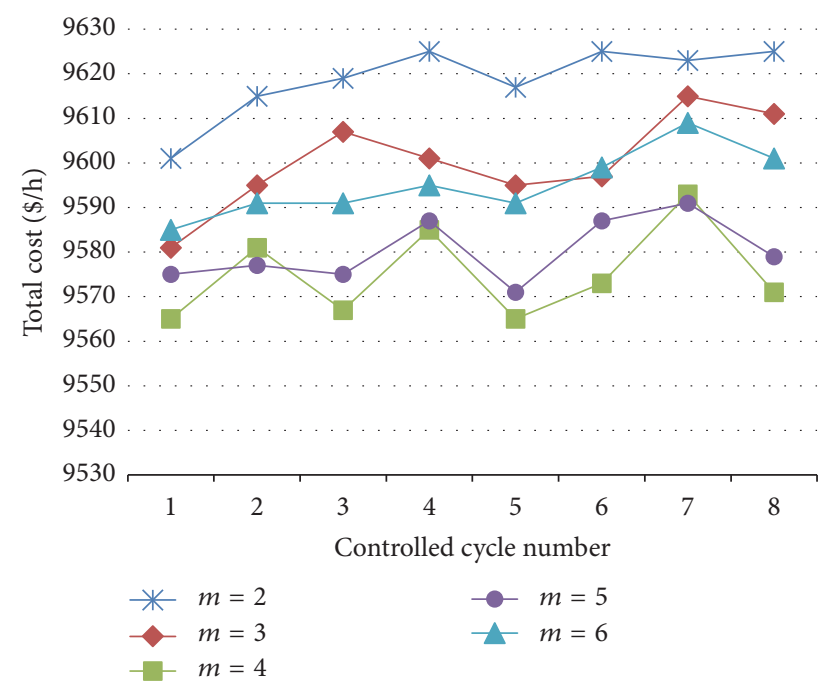

Figure 7: Total cost of controlled cycle with different $m$ value.

an integrated strategy can minimize the total cost, of a transit service, and the optimal scheme will be achieved when $m=4$. Thus, assuming that the computation time in this paper is adequate, the number of controlled vehicle trips at any given time is equal to 4 .

\section{Results}

To evaluate the performance of our proposed integrated strategy, we test three alternative strategies: (1) normal operation (with no controls), (2) limited-stop only strategy (without short-turning), and (3) an integrated limited-stop and shortturn strategy with turn-back holding. Under the normal operation, buses would stop at every station and run between the terminals of bus route without short-turning service. The limited-stop only strategy not only makes buses operate along the entire route, but also allows buses to skip several stations. Under the integrated limited-stop and short-turn strategy with turn-back holding, buses do not have to serve the entire route and will turn back halfway. Meanwhile, the integrated strategy also allows buses to skip several stations during serving segments. These three strategies are made to compare the effectiveness of each. In the case of the normal operation strategy, buses serve all stations on the route; each $y_{i, j}$ is equal to 1 . Using the limited-stop only strategy (without shortturning), we only need to search the $y_{i, j}$ scheme. Also, as with the normal operation strategy, the limited-stop only strategy does not consider $x_{i, j^{\prime}}$. The integrated strategy must adopt the above solution method, which searches $x_{i, j^{\prime}}$, determines the previous bus and the next bus of turn-back bus in the other direction under the initial scheme, calculates the turnback holding time, and then determines $y_{i, j}$. Under the same conditions, the parameters in the integrated strategy are the same as those in the other two strategies. The results shown in Figure 8 present the total cost per hour under all three strategies.

Obviously, the integrated strategy has the best effect on the transit system, compared to the normal operation strategy



Figure 8: Total cost of controlled cycle under three strategies.

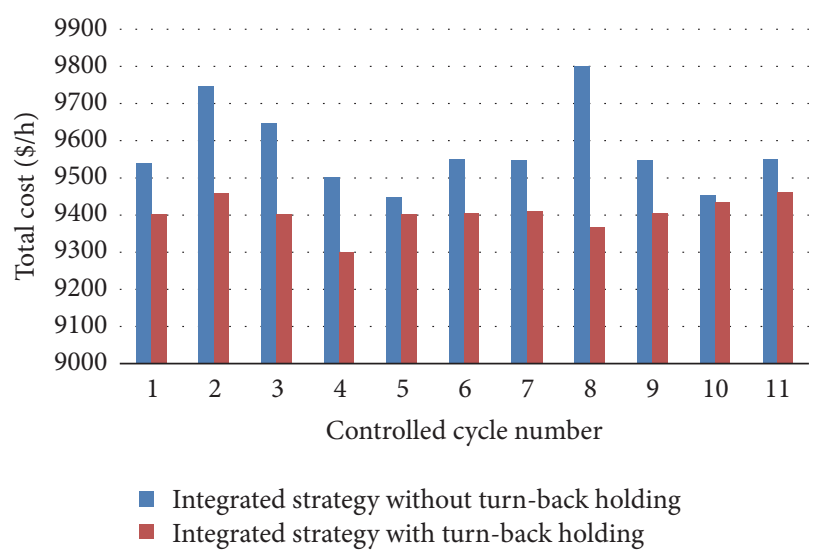

FIGURE 9: The influence of turn-back holding on the total cost.

and the limited-stop strategy without short-turning strategy. Compared with the normal operation strategy, the limitedstop only without short-turning strategy can reduce the total cost by $\$ 256$ per hour, while the total cost can be reduced by $\$ 914$ per hour by the integrated strategy. There is no doubt that the integrated limited-stop and short-turn strategy can save more in terms of cost than the limited-stop only without short-turning strategy. Meanwhile, only the limitedstop strategy can decrease the bus running time and the passenger in-vehicle time, but this strategy also increases passenger waiting time. In addition, the integrated limitedstop and short-turn strategy can greatly reduce bus running time, allowing the total cost to be significantly decreased.

What is more, the influence of turn-back holding on the total cost is analyzed and shown in Figure 9. Figure 9 shows that turn-back holding has a significant influence on bus routes. The integrated strategy without turn-back holding may actually disturb the effective operation of buses, decrease transit service reliability, and also increase passenger waiting time. Obviously, then, implementing turn-back holding is a valid and necessary means by which to improve a transit service and reduce the total cost of running that service for both the service provider and the passenger. 


\section{Conclusions}

In this paper, we implement a real-time integrated limitedstop and short-turning bus route strategy. Also, by considering stochastic travel time, our integrated strategy has the objective of improving the quality and cost of unbalanced and unreliable transit services. In our strategy, after departing their original station, designated buses do not serve all stations on the route. Buses may skip those stations where passenger demand is low. In addition, some buses do not need to arrive at the bus route's normal terminal station. These buses can turn back at appropriate stations, in order to reduce running time. In order to avoid disorderly bus operations after turn-backs, the practice of bus holding is adopted at the turn-back station. From our test, several conclusions can be found, as follows.

Through an examination to determine the optimum number of controlled vehicle trips at any given time, we can see that a change of $m$ will have an influence on the objective formulation. If the number of controlled vehicles is too small or too large, the objective of balancing passenger demand and improving the timeliness of the real-time strategy cannot be achieved.

Compared with the normal operation and the limitedstop only without short-turning strategy, our integrated strategy can save the most in terms of the total cost of a transit system. The limited-stop and short-turning strategies can reduce bus running time and passenger in-vehicle time, but these strategies will also increase passenger waiting time.

Finally, with the objective of ensuring that buses operate regularly, the practice of bus holding at turn-back stations is put in place and can be a valid means to save regarding the total transit service cost.

\section{Notations}

$i$ : Index of vehicles, $i=0,1,2, \ldots$

$j: \quad$ Index of stations, $j=1,2, \ldots, n$

$t_{i, j}^{A}: \quad$ Arrival time of bus $i$ at station $j$

$t_{i, j}^{D}$ : $\quad$ Departure time of bus $i$ at station $j$

$t_{i, j}^{E}$ : Dwelling time of bus $i$ at station $j$

$t_{\mathrm{ac}}$ : Bus acceleration time

$t_{j-1, j}^{R}$ : Running time between station $j-1$ and station $j$

$t_{\mathrm{de}}: \quad$ Bus deceleration time

$H_{\text {min }}$ : Minimum headway

$x_{i, j^{\prime}}$ : Variable that indicates the type of station $j^{\prime}$ for bus $i$; if bus $i$ turns back at station $j^{\prime}$, then $x_{i, j^{\prime}}=1$; if not, $x_{i, j^{\prime}}=0$

$y_{i, j}$ : Variable that indicates the type of station $j$ for bus $i$; if bus $i$ serves station $j$, then $y_{i, j}=1$; if bus $i$ skips station $j$, then $y_{i, j}=0$

$j^{\prime}$ : Turn-back station in the one direction, and $N-j^{\prime}+1$ is the turn-back station in the other station

$t_{i, j^{\prime}}^{h}$ : Bus holding time for the turn-back bus at station $j^{\prime}$

$\alpha$ : $\quad$ Average passenger boarding time $\beta$ : $\quad$ Average passenger alighting time

$N_{i, j}^{+}$: Number of boarding passengers riding bus $i$ at station $j$

$N_{i, j}^{-}: \quad$ Number of alighting passengers riding bus $i$ at station $j$

$\lambda_{i, j \rightarrow k}$ : Arrival rate of passengers from station $j$ to station $k$

$W_{i, j}: \quad$ Average waiting time of passengers for bus $i$ at station $j$

$c_{b}$ : $\quad$ Running time value

$c_{w}: \quad$ Waiting time value

$c_{i}$ : In-vehicle time value.

\section{Conflicts of Interest}

The authors declare that they have no conflicts of interest.

\section{Acknowledgments}

This work was supported by the National Natural Science Foundation of China (Grant no. 51378237) and the National Science Foundation for Young Scientists of China (Grant no. 51608224).

\section{References}

[1] M. A. Turnquist and S. W. Blume, "Evaluating potential effectiveness of headway control strategies for transit systems," Transportation Research Record, no. 746, pp. 25-29, 1980.

[2] M. D. Abkowitz and M. Lepofsky, "Implementing headwaybased reliability control on transit routes," Journal of Transportation Engineering, vol. 116, no. 1, pp. 49-63, 1990.

[3] X. J. Eberlein, N. H. M. Wilson, and D. Bernstein, "The holding problem with real-time information available," Transportation Science, vol. 35, no. 1, pp. 1-18, 2001.

[4] L. Fu and X. Yang, "Design and implementation of bus-holding control strategies with real-time information," Transportation Research Record, vol. 1791, pp. 6-12, 2002.

[5] F. Delgado, J. C. Munoz, and R. Giesen, "How much can holding and/or limiting boarding improve transit performance?" Transportation Research Part B: Methodological, vol. 46, no. 9, pp. 1202-1217, 2012.

[6] C. Leiva, J. C. Muñoz, R. Giesen, and H. Larrain, "Design of limited-stop services for an urban bus corridor with capacity constraints," Transportation Research Part B: Methodological, vol. 44, no. 10, pp. 1186-1201, 2010.

[7] C. E. Cortés, S. Jara-Díaz, and A. Tirachini, "Integrating short turning and deadheading in the optimization of transit services," Transportation Research Part A: Policy and Practice, vol. 45, no. 5, pp. 419-434, 2011.

[8] J. Chen, Z. Liu, S. Zhu, and W. Wang, "Design of limited-stop bus service with capacity constraint and stochastic travel time," Transportation Research Part E: Logistics and Transportation Review, vol. 83, pp. 1-15, 2015.

[9] W. Suh, K.-S. Chon, and S.-M. Rhee, "Effect of skip-stop policy on a Korean subway system," Transportation Research Record, no. 1793, pp. 33-39, 2002.

[10] L. Fu, Q. Liu, and P. Calamai, "Real-time optimization model for dynamic scheduling of transit operations," Transportation Research Record, no. 1857, pp. 48-55, 2003. 
[11] A. Sun and M. Hickman, "The real-time stop-skipping problem," Journal of Intelligent Transportation Systems, vol. 9, no. 2, pp. 91-109, 2005.

[12] M. M. O. Sidi, S. Hammadi, S. Hayat, and P. Borne, "Urban transport network regulation and evaluation: A fuzzy evolutionary approach," IEEE Transactions on Systems, Man, and Cybernetics Part A: Systems and Humans, vol. 38, no. 2, pp. 309318, 2008.

[13] C. E. Cortés, D. Sáez, F. Milla, A. Núñez, and M. Riquelme, "Hybrid predictive control for real-time optimization of public transport systems' operations based on evolutionary multiobjective optimization," Transportation Research Part C: Emerging Technologies, vol. 18, no. 5, pp. 757-769, 2010.

[14] D. Sáez, C. E. Cortés, F. Milla, A. Núñez, A. Tirachini, and M. Riquelme, "Hybrid predictive control strategy for a public transport system with uncertain demand," Transportmetrica, vol. 8, no. 1, pp. 61-86, 2012.

[15] B. Yu, Z. Z. Yang, and S. Li, "Real-time partway deadheading strategy based on transit service reliability assessment," Transportation Research A: Policy and Practice, vol. 46, no. 8, pp. 12651279, 2012.

[16] J. C. Muñoz, C. E. Cortés, R. Giesen et al., "Comparison of dynamic control strategies for transit operations," Transportation Research Part C: Emerging Technologies, vol. 28, pp. 101-113, 2013.

[17] J. E. Xu, N. H. M. Wilson, C. Barnhart, and D. Bernstein, "The real-time deadheading problem in transit operations control," Transportation Research B: Methodological, vol. 32, no. 2, pp. 77100, 1998 .

[18] S. Chuanjiao, Scheduling optimization of bus rapid transit, Chang'an University, 2008. 


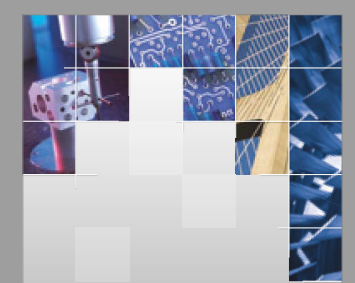

\section{Enfincering}
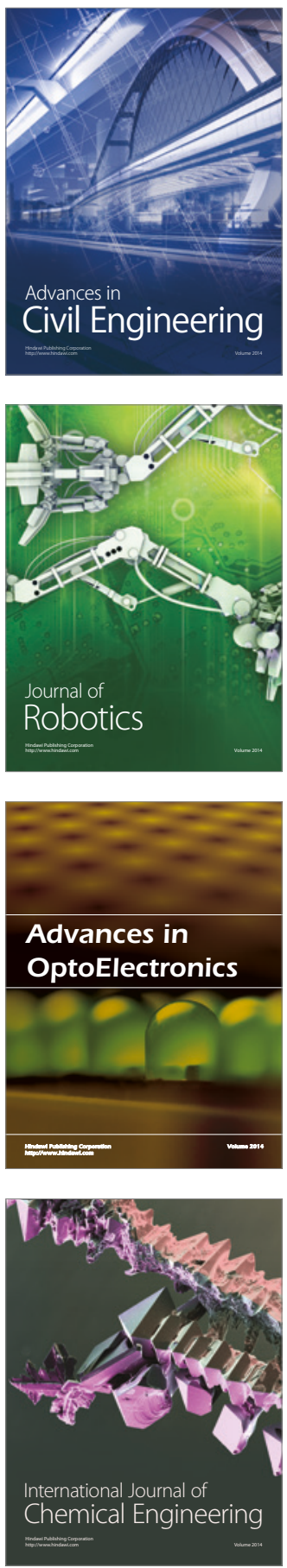

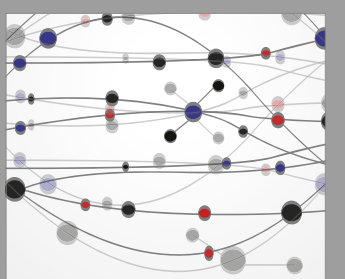

The Scientific World Journal

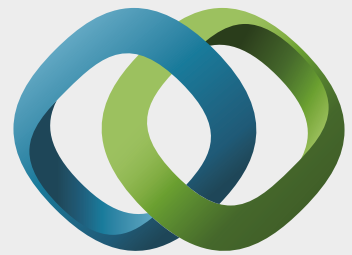

\section{Hindawi}

Submit your manuscripts at

https://www.hindawi.com
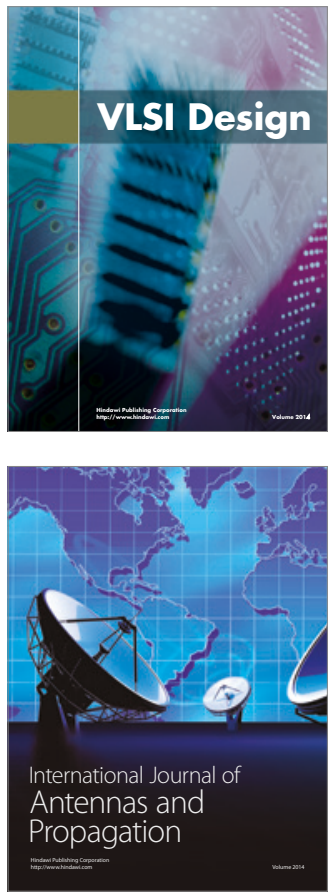

\section{Rotating}

Machinery
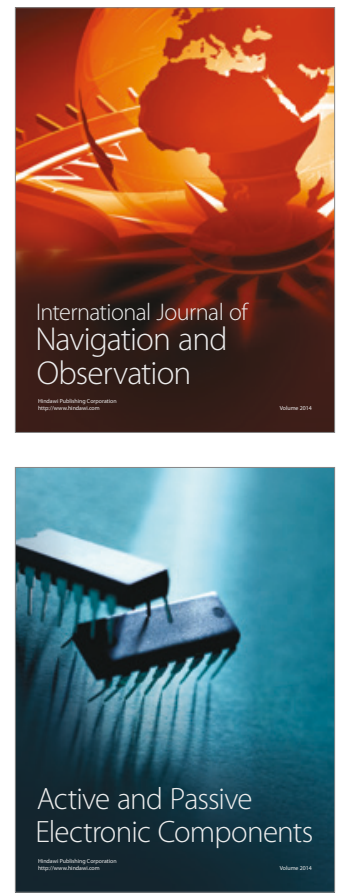
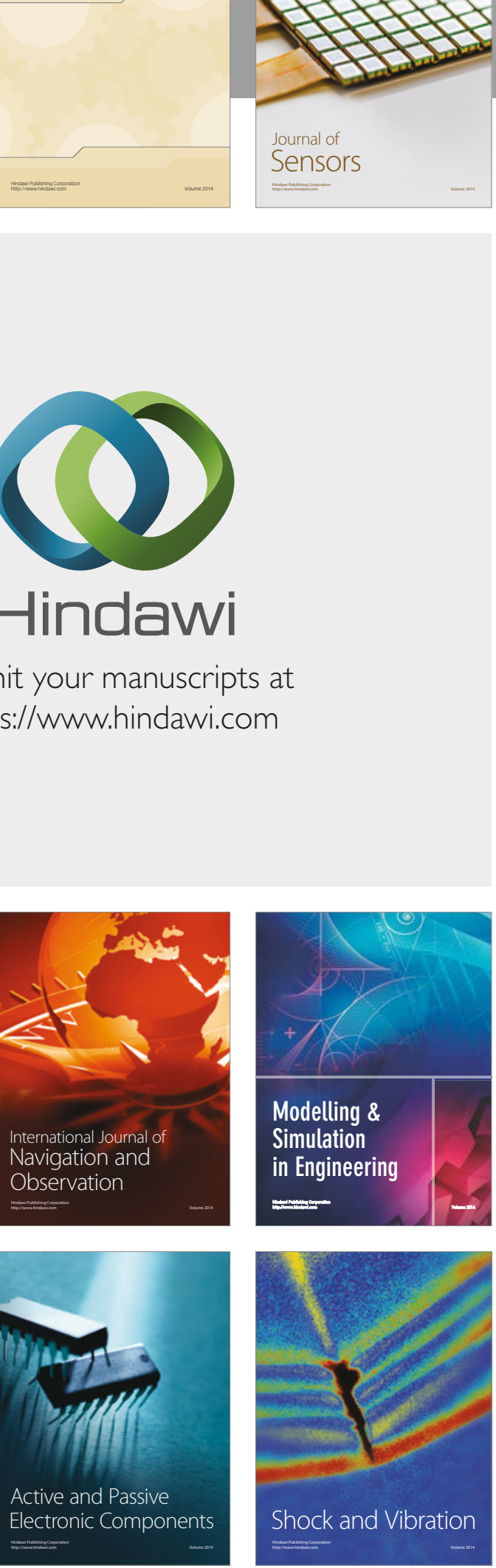
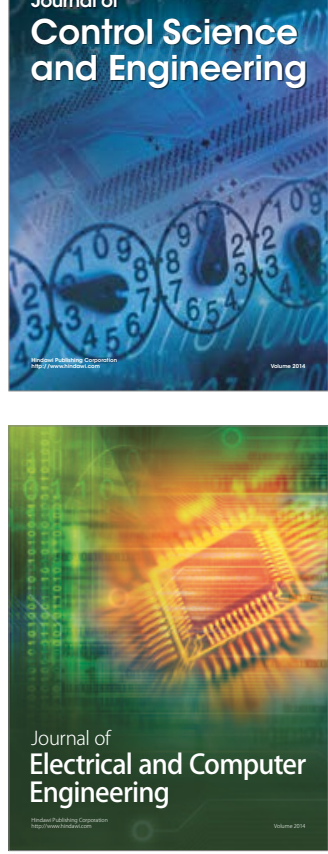

Distributed

Journal of

Control Science

and Engineering
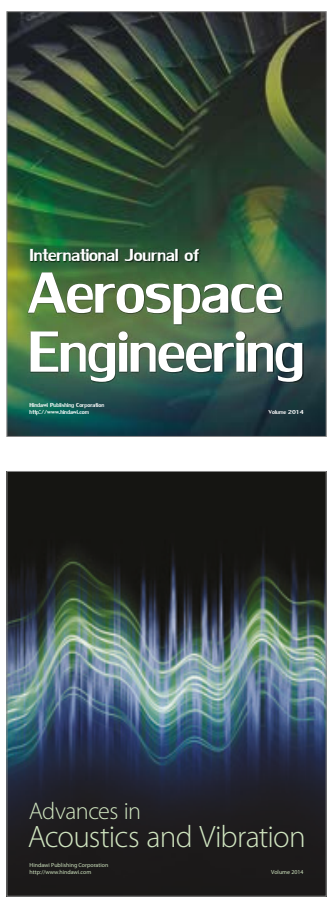

Sensor Networks 